\title{
Sediment and Cavitation Erosion Studies through Dam Tunnels
}

\author{
Muhammad Abid, ${ }^{1}$ Jamal Saeed, ${ }^{2}$ and Hafiz Abdul Wajid ${ }^{3,4}$ \\ ${ }^{1}$ Interdisciplinary Research Center, COMSATS Institute of Information Technology, Wah Cantonment 47040, Pakistan \\ ${ }^{2}$ School of Mechanical and Manufacturing Engineering, NUST, Islamabad 44000, Pakistan \\ ${ }^{3}$ Department of Mathematics, COMSATS Institute of Information Technology, Lahore 54000, Pakistan \\ ${ }^{4}$ Department of Electrical Engineering, Islamic University of Madinah, Madinah 20012, Saudi Arabia \\ Correspondence should be addressed to Muhammad Abid; drabid@ciitwah.edu.pk
}

Received 6 November 2015; Accepted 21 February 2016

Academic Editor: Sheng-Rui Jian

Copyright (C) 2016 Muhammad Abid et al. This is an open access article distributed under the Creative Commons Attribution License, which permits unrestricted use, distribution, and reproduction in any medium, provided the original work is properly cited.

\begin{abstract}
This paper presents results of sediment and cavitation erosion through Tunnel 2 and Tunnel 3 of Tarbela Dam in Pakistan. Main bend and main branch of Tunnel 2 and outlet 1 and outlet 3 of Tunnel 3 are concluded to be critical for cavitation and sediment erosion. Studies are also performed for increased sediments flow rate, concluding $5 \mathrm{~kg} / \mathrm{sec}$ as the critical value for sudden increase in erosion rate density. Erosion rate is concluded to be the function of sediment flow rate and head condition. Particulate mass presently observed is reasonably low, hence presently not affecting the velocity and the flow field.
\end{abstract}

\section{Introduction}

In recent studies, it is highlighted that sediments are gradually accumulated in Tarbela Dam reservoir, resulting in gradual decrease in reservoir water storage capacity, increased load on embankment wall, and damage to tunnels and turbines [1-3]. Among the tunnels of Tarbela Dam, Tunnel 2 and Tunnel 3 are observed to be the most critical [3, 4]; their parameters are given in Table 1 . Tunnel 3 is a bigger tunnel with horizontal inlet at the reservoir bed and intake of $2415.64 \mathrm{~m}^{3} / \mathrm{sec}$ whereas Tunnel 2 has vertical inlet and intake of $978.63 \mathrm{~m}^{3} / \mathrm{sec}$. Both tunnels are used for irrigation and power generation purposes. Abid et al. in [4-7] have highlighted erosion of the walls of tunnels with present sediment flow rate through them. As sediment accumulation is increased, the number of sediments particles also changes through the tunnels; hence their effect on the tunnels life needs to be investigated. Reynolds Stress Model (RSM) due to its advantage of performing well in highly chaotic and swirling flows and uneven geometries is used to predict flow separation [8]. Continuity and modified Navier Stokes equation [1-3] shows Reynolds' Stress $\sigma$ which is a flow property and is taken as zero in nonturbulent flows.
The constant and coefficients in the RSM used are given in Table 2. Hence,

$$
\begin{gathered}
\frac{\partial \rho}{\partial t}+\nabla \cdot\left(\rho U_{c}\right)=0, \\
\frac{\partial\left(\rho U_{c}\right)}{\partial t}+\nabla \cdot\left(\rho U_{c} \otimes U_{c}\right)=B+\nabla \cdot\left(-\rho u^{\prime} \otimes u^{\prime}\right)+\sigma,
\end{gathered}
$$

where

$$
\sigma=\frac{p}{\rho} I+\frac{\mu}{\rho}\left[\nabla U_{c}+\left(\nabla U_{c}\right)^{T}\right] .
$$

Lagrangian particle tracking is used as particles experience a number of forces while passing through the domain including buoyancy, lift, drag, and weight. However, buoyancy, for particles having high density, is neglected as this force is negligible. The drag force thus experienced by a particle is calculated using carrier velocity $U_{c}$ and particle velocity $U_{p}$ as per relation in

$$
\frac{d U_{p}}{d x}=F_{D}\left(U_{c}-U_{p}\right) .
$$


TABLE 1: Tunnels parameters.

\begin{tabular}{lcc}
\hline Parameters & Tunnel 2 & Tunnel 3 \\
\hline Length (m) & 846.51 & 907.41 \\
Inlet Elevation (m) & 373 & 360.43 \\
Outlet Elevation (m) & 337.11 & 340.46 \\
Inlet Diameter (m) & 10.96 & 48.87 \\
Outlet Branches Diameter (m) & 4.87 & 7.32 \\
Average Volume Flow Rate of Water $\left(\mathrm{m}^{3} / \mathrm{sec}\right)$ & 978.63 & 2415.64 \\
Average Available Head (m) & 950.91 & 918.15 \\
Power Generation Capacity (MW) & 1050 & 1728 \\
\hline
\end{tabular}

TABLE 2: Constant and coefficients used in RS Model.

\begin{tabular}{lll}
\hline Anisotropic Diffusion Constant & $C_{\mu}$ & 0.09 \\
\hline Turbulent Schmidt Number & $\sigma_{k}$ & 1.00 \\
\hline & $C_{s 1}$ & 1.80 \\
Reynolds' Stress Coefficients & $C_{s 2}$ & 0.60 \\
& $C_{1 \varepsilon}$ & 1.44 \\
& $C_{2 \varepsilon}$ & 1.92 \\
\hline
\end{tabular}

The drag force per unit mass $F_{D}$ of the particle is calculated using (4), where $C_{D}$ is the Drag Coefficient and is taken as 0.44 for the two-way coupling considered:

$$
F_{D}=\frac{1}{\tau_{p}} C_{D} \frac{\mathrm{Re}_{p}}{24} .
$$

Particle's response time to change in flow and particles Reynolds' number are calculated using (5) and (6), respectively. These equations are given as follows:

$$
\begin{aligned}
\tau_{p} & =\frac{\rho_{p} d_{p}^{2}}{18 \mu}, \\
\operatorname{Re}_{p} & =\frac{d_{p}\left(U-U_{p}\right)}{v},
\end{aligned}
$$

where $U$ is the carrier phase velocity while $U_{p}$ is the particle velocity and $v$ is the carrier phase dynamic viscosity.

Finnie erosion model given in (7) is used in conjunction with Lagrangian particle tracking in $\operatorname{ANSYS~CFX~}^{\circledR}[8,9]$. Water passing through the tunnels carries sediment particles and in the regions where there is discontinuity in the direction of flow or turbulence, particles disassociate themselves from the water and follow a path dictated by its inertia because of high Stokes number [10]. In these regions, particles strike the walls of the tunnels resulting in erosion. Erosion due to sediment particles is a function of impact velocity and impact angle of the particles. The exponent $n$ in the Finnie erosion model is taken as 2 and $k$ is taken as 1. Two-way coupling or multiphase flow conditions are considered as the effect of increased number of sediment particles for erosion is studied [10]. Hence,

$$
E=k \cdot V_{p}^{n} \cdot f(\gamma)
$$

TABLE 3: Variables used in Rayleigh-Plesset's cavitation model.

\begin{tabular}{lc}
\hline$P_{s}$ & $4240 \mathrm{~Pa}$ \\
$F_{\text {cond }}$ & 0.01 \\
$F_{\text {vap }}$ & 50 \\
$\rho_{r}$ & 1000 \\
$r_{\text {nuc }}$ & $5 e-4$ \\
$R_{f}$ & 0.25 \\
$R_{B}$ & $1 \mu \mathrm{m}$ \\
\hline
\end{tabular}

where $f(\gamma)=(1 / 3) \cos ^{2}(\gamma)$ when $\tan (\gamma)>1 / 3$ and $f(\gamma)=$ $\sin (\gamma)-3 \sin ^{2}(\gamma)$ when $\tan (\gamma)<1 / 3$.

Cavitation is a phenomenon that results from a pressure drop of the liquid phase below saturation pressure of the liquid under the conditions. Based on the tunnels geometry, $\mathrm{S}$ bend and outlet branches sharp bends, pressure drop of water is expected along these locations. Therefore, erosion due to cavitation phenomenon is studied. Rayleigh-Plesset's model given in (8) is used to govern the water vapors formation and condensation [11] and different variables used in the model are summarized in Table 3. Therefore,

$$
R_{B} \frac{d^{2} R_{B}}{d t^{2}}+1.5\left(\frac{d R_{B}}{d t}\right)^{2}+\frac{2 \sigma}{\rho_{f} R_{B}}=\frac{p_{v}-p}{\rho_{f}}
$$

where $R_{B}$ is nucleation radius of the bubble, $p_{v}$ is vapor pressure, $p$ is reference pressure, and $\rho_{f}$ is the fluid density.

\section{Modeling, Meshing, and Boundary Conditions}

Both tunnels are modeled in Pro-Engineer software [12] (Figure 1) and mesh is generated in ANSYS ICEM CFD ${ }^{\circledR}$ [8]. In order to capture erosion along inner walls, a higher number of elements with prism elements are added in finite element model of Abid et al. [3-7] (Figure 2). Table 4 shows results of mesh sensitivity analysis including number and size of elements, computational time, and other variables [13]. Table 5 shows general CFD parameters used in ANSYS CFX ${ }^{\circledR}$. Table 6 shows boundary conditions, initialization condition, and hypothetical sediment flow rate in the tunnels to study the effect of increased number of particles on erosion rate density during winter, average, and summer seasons, that is, low, medium, and high water heads. To save computational time and resource, velocities have been initialized to get converged solution sooner. Particle injection is considered uniform based on the geometry of the tunnels.

\section{Results and Discussion}

The velocity of water at different critical locations of Tunnel 2 and Tunnel 3 for different head condition and sediments flow rate is summarized in Tables 7 and 8, respectively. No change in velocity of the water with variation in sediments flow rate is observed and is concluded due to small particulate mass, hence no effect on the flow field. Along different tunnel sections, different velocities are recorded. Maximum and 


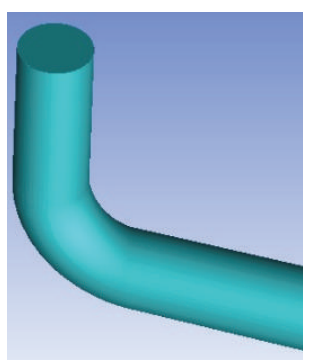

(a)

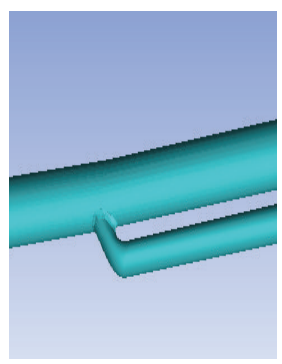

(b)

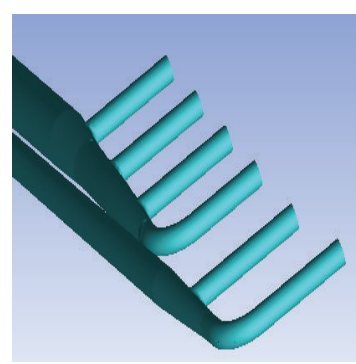

(c)

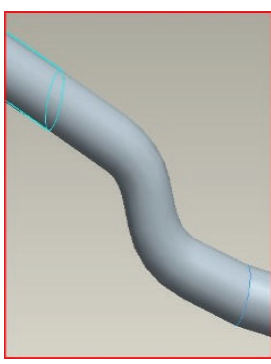

(d)

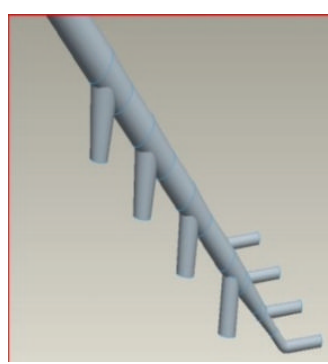

(e)

FIgURE 1: Modeling of Tunnel 2: (a) main bend, (b) main branch, (c) outlets: Tunnel 3, (d) S bend, and (e) outlets.

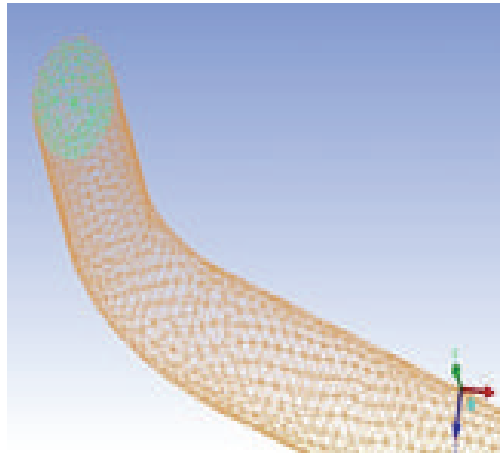

(a)

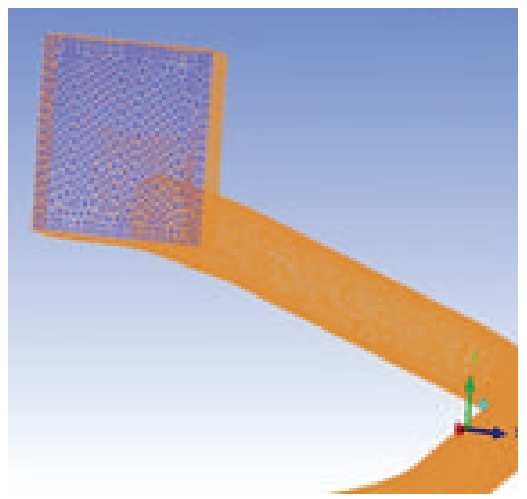

(d)

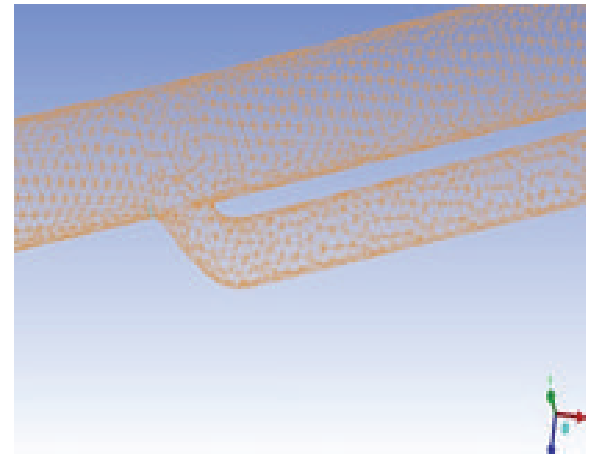

(b)

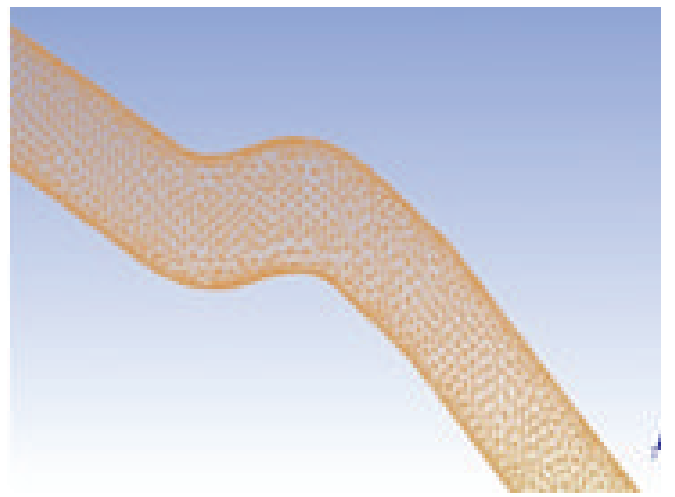

(e)

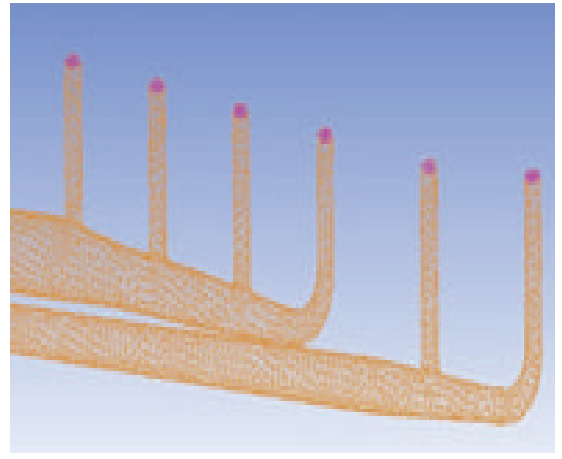

(c)

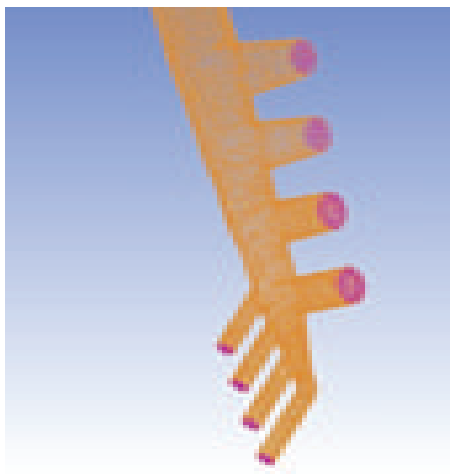

(f)

Figure 2: Meshing: Tunnel $2(\mathrm{a}-\mathrm{c})$ and Tunnel $3(\mathrm{~d}-\mathrm{f})$.

minimum velocity are observed at maximum and minimum water heads, respectively. It is also concluded that any change in water velocity results in change in sediment velocity.

Erosion rate density is observed to be increased with increase in sediment flow rate. For high head condition, the change can be easily attributed to the increase in velocities at all critical locations of both tunnels. Figures 3 and 4 show changes in erosion rate for Tunnel 2 and Tunnel 3, respectively, with change in sediment flow rate at different head conditions. As velocity does not change at any location under the same head condition for different sediments flow rate, the minor variation in erosion rate density is concluded due to the slight variation in impact angle. For both tunnels, until $5 \mathrm{~kg} / \mathrm{sec}$ sediment flow rate, almost zero erosion rate density is observed which however started increasing rapidly after this and became prominent at the sediment flow rate of $50 \mathrm{~kg} / \mathrm{sec}$. This concludes that the sediment flow rate should be carefully measured to avoid any catastrophic failure of the tunnels. Main branch of Tunnel 2 and outlet 3 of Tunnel 3 are concluded to be critical for sediment erosion. Results for sediment erosion density rate for Tunnel 2 and Tunnel 3 are summarized in Tables 9 and 10, respectively.

It is observed from water and sediment flow through the tunnels that pressure at the inside of the bends or sharp corners drops below saturation pressure resulting in water vapors formation. Analyses were performed for the various 
TABLE 4: Mesh sensitivity analysis.

\begin{tabular}{|c|c|c|c|c|}
\hline \multirow{2}{*}{ Equations } & \multicolumn{4}{|c|}{ Mesh size } \\
\hline & 1 & 2 & 3 & 5 \\
\hline U-Momentum Bulk & $1.06 E-04$ & $1.42 E-04$ & $1.66 E-04$ & $2.46 E-04$ \\
\hline V-Momentum Bulk & $5.56 E-05$ & $5.35 E-05$ & $4.46 E-05$ & $3.49 E-05$ \\
\hline W-Momentum Bulk & $7.62 E-05$ & $7.99 E-05$ & $5.68 E-05$ & $8.05 E-05$ \\
\hline Mass of Water & $1.19 E-05$ & $1.72 E-05$ & $1.87 E-05$ & $2.47 E-05$ \\
\hline uu-RS & $5.12 E-04$ & $4.47 E-04$ & $9.12 E-04$ & $1.00 E-03$ \\
\hline vv-RS & $6.16 E-04$ & $4.06 E-04$ & $5.19 E-04$ & $4.16 E-04$ \\
\hline ww-RS & $1.03 E-03$ & $4.98 E-04$ & $4.98 E-04$ & $4.67 E-04$ \\
\hline uv-RS & $9.94 E-05$ & $5.43 E-05$ & $1.96 E-04$ & $2.49 E-04$ \\
\hline uw-RS & $1.90 E-04$ & $2.54 E-04$ & $1.91 E-04$ & $2.04 E-04$ \\
\hline vw-RS & $2.71 E-04$ & $1.19 E-04$ & $6.00 E-05$ & $6.25 E-05$ \\
\hline E-Dissipation K & $1.71 E-04$ & $1.20 E-04$ & $9.42 E-05$ & $4.88 E-05$ \\
\hline Computational time (sec) & 4310 & 1900 & 1104 & 1623 \\
\hline
\end{tabular}

TABLE 5: Various constants and coefficients used in simulation.

\begin{tabular}{lccc}
\hline Parameter & Detail & Tunnel 2 & Tunnel 3 \\
\hline Erosion & Finnie Model & $k=1, n=2$ & $k=1, n=2$ \\
Particles injection & Uniform injection & Two-way coupled & Two-way coupled \\
Restitution Coefficient & Perpendicular and parallel & 0.9 and 1, respectively & 0.9 and 1, respectively \\
Drag Coefficient & Schiller and Neumann Correlation & 0.44 for Re f $_{d}>1000$ & 0.44 for Re f $_{d}>1000$ \\
Particle Integration & Tracking distance and time & $1200 \mathrm{~m}, 300 \mathrm{sec}$ & $1200 \mathrm{~m}, 300 \mathrm{sec}$ \\
\hline
\end{tabular}

TABLE 6: Boundary conditions, initialization condition, and sediment flow rates at different heads.

\begin{tabular}{|c|c|c|c|c|}
\hline & Type & Head & Tunnel 2 & Tunnel 3 \\
\hline \multirow{3}{*}{ Boundary conditions } & \multirow{3}{*}{ Pressure $(\mathrm{kPa})$} & High & 1323.53 & 1290.76 \\
\hline & & Medium & 950.91 & 918.15 \\
\hline & & Low & 578.30 & 545.53 \\
\hline \multirow{3}{*}{ Initial conditions } & \multirow{3}{*}{ Velocity $(\mathrm{m} / \mathrm{sec})$} & High & 11.55 & 2.05 \\
\hline & & Medium & 10.33 & 1.70 \\
\hline & & Low & 7.57 & 1.31 \\
\hline Sediment flow rates at & \multicolumn{4}{|c|}{$5 \times 10^{-5}, 5 \times 10^{-4}, 5 \times 10^{-3}, 5 \times 10^{-2}, 5 \times 10^{-1}, 5$, and 50} \\
\hline
\end{tabular}

TABLE 7: Velocity at different locations of Tunnel 2 under different head and varying sediment flow rates.

\begin{tabular}{ccccccccccc}
\hline Head & Sediment flow rate $(\mathrm{kg} / \mathrm{sec})$ & Main bend & Main branch & Out1 & Out2 & Out3 & Out4 & Out5 & Out6 \\
\hline \multirow{2}{*}{ High } & 0.00005 & 41.4 & 54.8 & 38.7 & 17.0 & 20.4 & 32.9 & 43.7 & 30.2 \\
& 50 & 41.4 & 54.8 & 38.7 & 17.0 & 20.4 & 32.9 & 43.7 & 30.2 \\
\hline \multirow{2}{*}{ Low } & 0.00005 & 31.0 & 41.0 & 29.0 & 12.8 & 15.3 & 24.6 & 32.8 & 22.6 \\
& 50 & 31.0 & 41.0 & 29.0 & 12.8 & 15.3 & 24.6 & 32.8 & 22.6 \\
\hline
\end{tabular}

TABLE 8: Velocity at different locations of Tunnel 3 under different head and varying sediment flow rates.

\begin{tabular}{lccccccc}
\hline Head & Sediment flow rate $(\mathrm{kg} / \mathrm{sec})$ & S1 & S2 & Out1 & Out2 & Out3 & Out4 \\
\hline \multirow{2}{*}{ Medium } & 0.00005 & 30.4 & 25.0 & 22.5 & 19.9 & 20.9 & 26.5 \\
& 50 & 30.4 & 25.0 & 22.5 & 19.9 & 20.9 & 26.5 \\
\hline \multirow{2}{*}{ Low } & 0.00005 & 22.4 & 18.4 & 16.6 & 14.7 & 15.5 & 19.5 \\
& 50 & 22.4 & 18.4 & 16.6 & 14.7 & 15.5 & 19.5 \\
\hline
\end{tabular}


TABLE 9: Erosion rate density $(\mathrm{kg} / \mathrm{m} \cdot \mathrm{sec})$ in Tunnel 2 under various head conditions and at fixed sediment flow rate of $50 \mathrm{~kg} / \mathrm{sec}$.

\begin{tabular}{lcccccccc}
\hline Head & Main bend & Main branch & Out1 & Out2 & Out3 & Out4 & Out5 & Out6 \\
\hline High & 45 & 324 & 269 & 34 & 49 & 52 & 62 & 107 \\
Medium & 28 & 237 & 198 & 30 & 38 & 34 & 60 & 62 \\
Low & 17 & 130 & 98 & 15 & 17 & 20 & 27 \\
\hline
\end{tabular}

TABLE 10: Erosion rate density $(\mathrm{kg} / \mathrm{m} \cdot \mathrm{sec})$ in Tunnel 3 under various head conditions and at fixed sediment flow rate of $50 \mathrm{~kg} / \mathrm{sec}$.

\begin{tabular}{lcccccc}
\hline Head & S1 & S2 & Out1 & Out2 & Out3 & Out4 \\
\hline High & 28 & 2.3 & 16 & 23 & 60 & 11 \\
Medium & 20 & 1.3 & 11 & 15 & 42 & 7 \\
Low & 12 & 1.0 & 7 & 8 & 24 & 2 \\
\hline
\end{tabular}

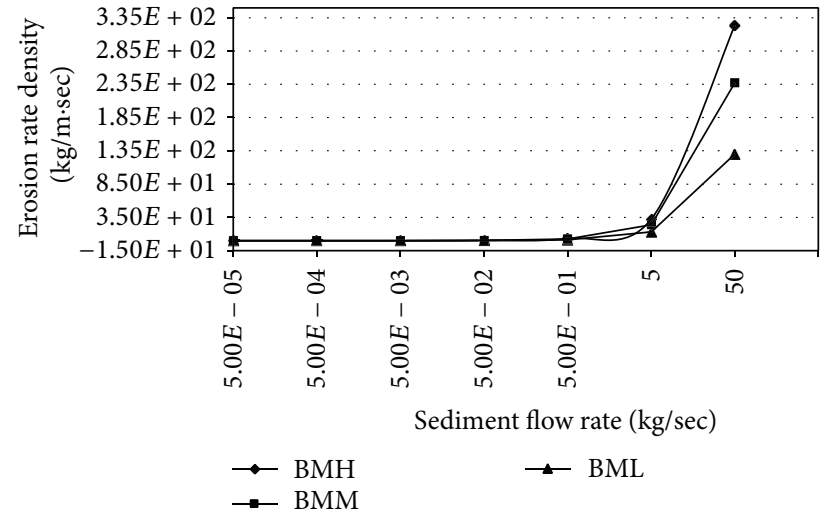

FIGURE 3: Erosion rate density at main branch for all head conditions and different sediment flow rate in Tunnel 2 (BM: main branch; $\mathrm{H}$ : high head; M: medium head; L: low head).

head conditions, that is, high, medium, and low. A significant pressure drop is observed at the main bend, main branch, and outlet branches in Tunnel 2 and $S$ bend and outlet branches in Tunnel 3. The volume fraction of water vapors is on the higher side at the critical locations of the tunnels, highlighting the notion that these locations are prone to erosion, and is concluded due to the cavitation effect. Cavitation erosion is therefore further superposed on the sediments erosion already observed. The presence of water vapors will bring these locations under a greater threat. The maximum water vapor volume fraction gradient, maximum volume fraction of water vapors, and Euler or cavitation numbers at different heads are summarized in Tables 11 and 12 for Tunnel 2 and Tunnel 3, respectively. It is concluded that the sharper bends have greater tendency of water vapors formation due to greater pressure drop at these locations. Critical locations are also identified based on the Euler or cavitation number calculated at these locations. Atmospheric pressure is taken to be the reference pressure. For Euler number less than 1, the region is termed "critical." Cavitation erosion is shown along

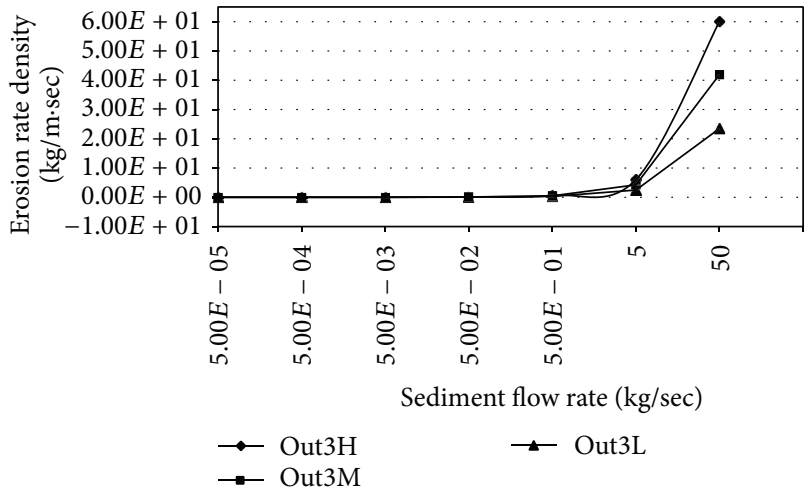

FIGURE 4: Erosion rate density at outlet 3 for all head conditions and different sediment flow rate in Tunnel 3 (Out: outlet; $\mathrm{H}$ : high head; M: medium head; L: low head).

different locations for Tunnel 2 and Tunnel 3 in Figures 5 and 6 , respectively. Main bend of Tunnel 2 and outlet 1 of Tunnel 3 are concluded to be critical for cavitation erosion.

\section{Conclusion}

Flow profile is observed to be not affected by the increase in sediment flow rate through the tunnels because of small particulate mass and negligible particle-to-particle interaction. The tracks followed by particles remained unchanged and any rise in erosion rate density is concluded as a direct consequence of head and sediment flow rate. Main branch of Tunnel 2 and outlet 3 of Tunnel 3 are concluded to be critical for sediment erosion.

Keeping in view the expected increased sediment flow rate in the tunnels due to sediment delta movement towards main embankment wall, for both tunnels, until $5 \mathrm{~kg} / \mathrm{sec}$ sediment flow rate, almost zero erosion rate density is observed which however started increasing rapidly after this and became prominent at the sediment flow rate of $50 \mathrm{~kg} / \mathrm{sec}$. 
TABLE 11: Volume fraction of water vapors and volume fraction gradient in Tunnel 2 under various head conditions.

\begin{tabular}{lccccc}
\hline Head & Main bend & Main branch & $\begin{array}{c}\text { Outlet branches } \\
\text { Outl Out6 }\end{array}$ & $\begin{array}{c}\text { Max. water vapor volume } \\
\text { fraction gradient }\left(\mathrm{m}^{-1}\right)\end{array}$ & $\begin{array}{c}\text { Max. volume fraction of } \\
\text { water vapors }\end{array}$ \\
\hline High & 0.75 & 0.98 & Volume fraction of water vapors and volume fraction gradient & 1.729 & 0.980 \\
Medium & 0.73 & 0.97 & 0.98 & 1.792 & 0.977 \\
Low & 0.72 & 0.96 & 0.97 & 1.764 & 0.967 \\
\hline & & & 0.96 & - \\
High & 0.09 & 0.24 & Euler or cavitation numbers & - \\
Medium & 0.43 & 1.11 & 0.13 & - \\
Low & 0.41 & 1.23 & 0.63 & - \\
\hline
\end{tabular}

TABLE 12: Volume fraction of water vapors, volume fraction gradient, and Euler or cavitation numbers in Tunnel 3 under various head conditions.

\begin{tabular}{lcccccccc}
\hline Head & S1 & S2 & Out1 & Out2 & Out3 & Out4 & $\begin{array}{c}\text { Max. water vapor volume } \\
\text { fraction gradient }\left(\mathrm{m}^{-1}\right)\end{array}$ & $\begin{array}{c}\text { Max. volume fraction of } \\
\text { water vapor }\end{array}$ \\
\hline High & 0.95 & 0.95 & 0.48 & 0.82 & 0.82 & 0.97 & 1.746 & 1.916 \\
Medium & 0.86 & 0.95 & 0.91 & 0.73 & 0.76 & 0.95 & 1.416 & 0.971 \\
Low & 0.87 & 0.72 & 0.67 & 0.45 & 0.57 & 0.74 & - & 0.906 \\
\hline & & & & & Euler or cavitation numbers & - \\
High & 0.86 & 1.09 & 0.41 & 0.39 & 0.51 & 0.96 & - & - \\
Medium & 0.97 & 1.12 & 0.49 & 0.68 & 0.80 & 1.12 & - \\
Low & 1.09 & 1.38 & 0.69 & 1.00 & 1.88 & 2.40 & -
\end{tabular}

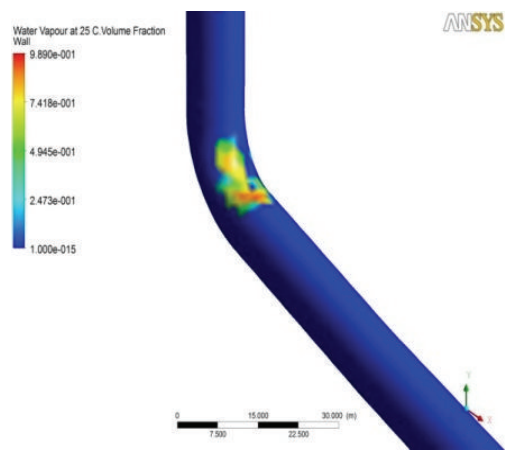

(a)

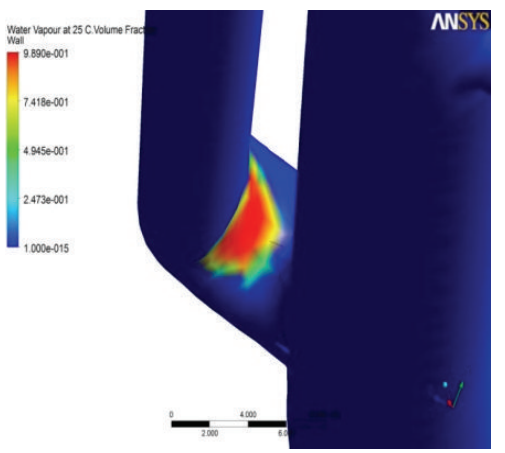

(b)

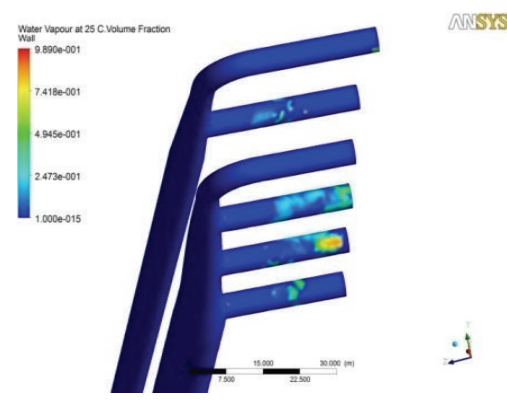

(c)

FIGURE 5: Cavitation erosion (water vapors) at different locations of Tunnel 2. 


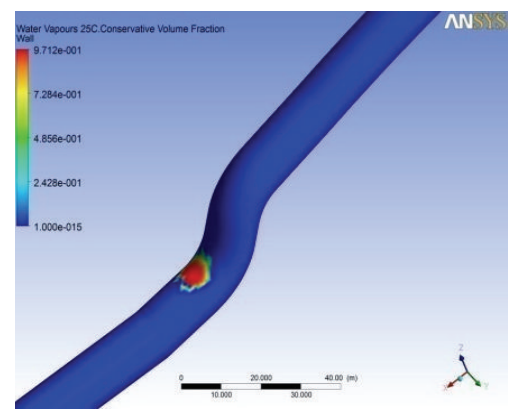

(a)

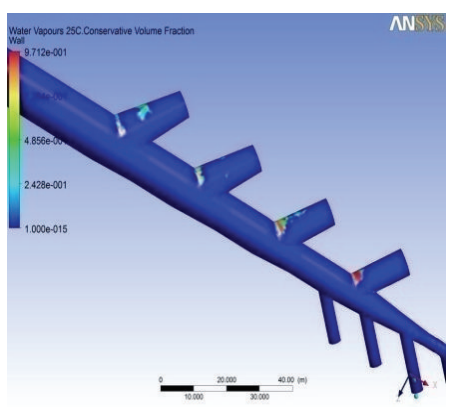

(b)

FIgURE 6: Cavitation erosion (water vapors) at different locations of Tunnel 3.

Hence, the possibility of catastrophic failure of the tunnels due to increased sediment flow rate cannot be ignored.

Cavitation is observed to be threatening at several locations. Main bend of Tunnel 2 and outlet 1 of Tunnel 3 are concluded to be critical for cavitation erosion. The combined effect of both erosion due to sediments and cavitation further increases the erosion rate density.

\section{Competing Interests}

The authors declare that they have no competing interests.

\section{References}

[1] M. Hanif, Sediment Concentration (ppm). Annual Reservoir Sedimentation Report, Survey and Hydrology Department, Tarbela Dam Project, 2009.

[2] M. Abid and M. U. Siddiqi, "Multiphase flow simulations through Tarbela Dam Spillways and Tunnels," Journal of Water Resource and Protection, vol. 2, no. 6, pp. 532-539, 2010.

[3] M. R. Siddiqui, Water and sediment flow simulation in tarbela dam reservoir [M.S. thesis], GIK Institute, Topi, Pakistan, 2010.

[4] A. A. Noon, Study of the effect of sediment flows through Tarbela Dam Tunnels [M.S. thesis], GIK Institute, Topi, Pakistan, 2010.

[5] M. Abid and A. A. Noon, "Turbulent flow simulations through Tarbela Dam Tunnel-2," Journal of Engineering, vol. 2, no. 7, pp. 205-213, 2010.

[6] M. Abid, A. A. Noon, and H. A. Wajid, "Simulation of turbulent flow through tarbela dam tunnel 3," IIUM Engineering Journal, vol. 11, no. 2, pp. 201-224, 2010.

[7] M. Abid, A. A. Noon, M. W. Al-Grafi, and H. A. Wajid, "Erosion study of Tarbela Dam Tunnel-1," Iranian Journal of Science and Technology, vol. 38, no. 1, pp. 253-261, 2014.

[8] ANSYS, NSYS CFX. Reference Guide, Release 11, ANSYS, 2009.

[9] I. Finnie, "Erosion of surfaces by solid particles," Wear, vol. 3, no. 2, pp. 87-103, 1960.

[10] G. Brown, "Use of CFD to predict and reduce erosion in industrial slurry piping system," in Proceedings of the 5th International Conference on CFD in the Process Industries (CSIRO '06), Melbourne, Australia, December 2006.

[11] J. Madadnia and I. Owen, "Accelerated surface erosion by cavitating particulate-laden flows," Wear, vol. 165, no. 1, pp. 113116, 1993.
[12] Pro/Engineer, Wildfire Release 4, Parametric Technology Corporation, 2009.

[13] G. Iaccarino, "Predictions of a turbulent separated flow using commercial CFD codes," Journal of Fluids Engineering, vol. 123, no. 4, pp. 819-828, 2001. 


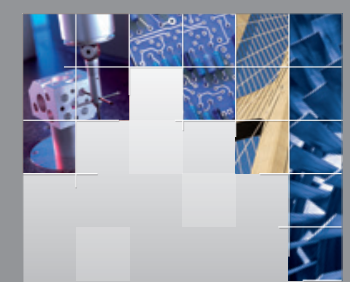

\section{Enfincering}
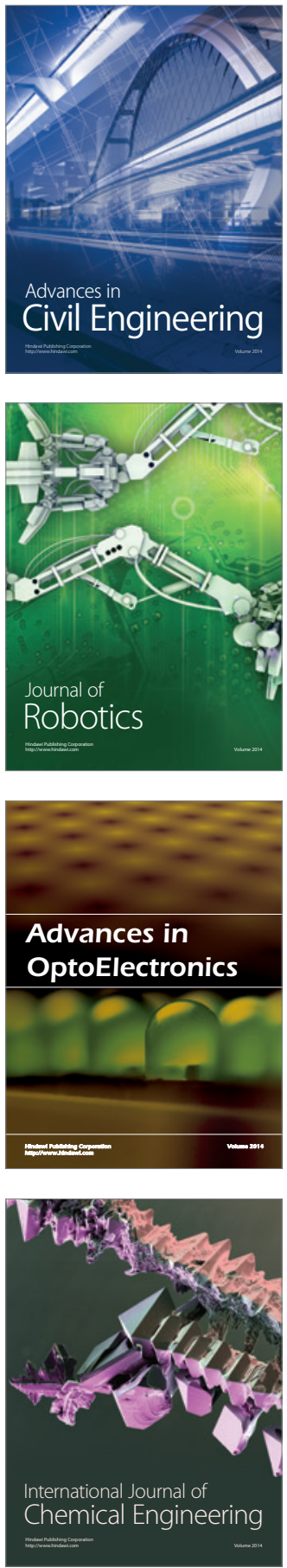

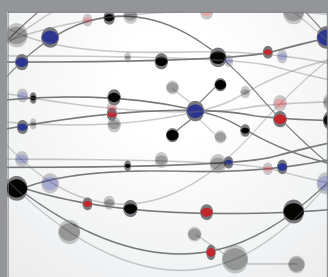

The Scientific World Journal

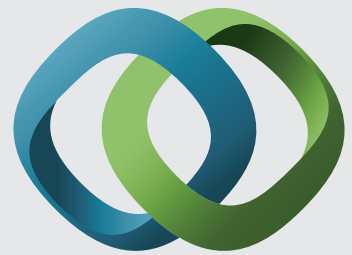

\section{Hindawi}

Submit your manuscripts at

http://www.hindawi.com
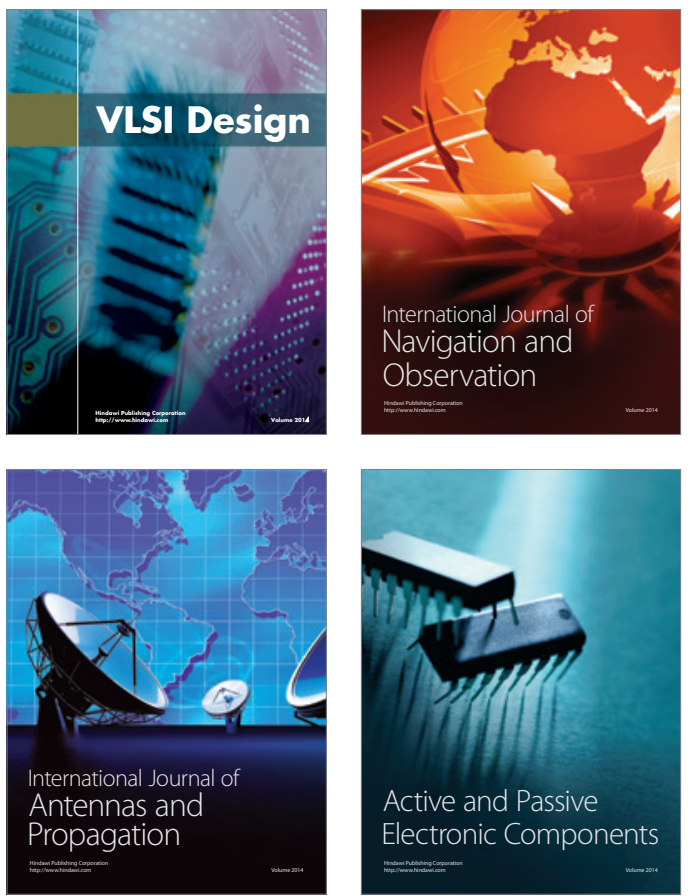
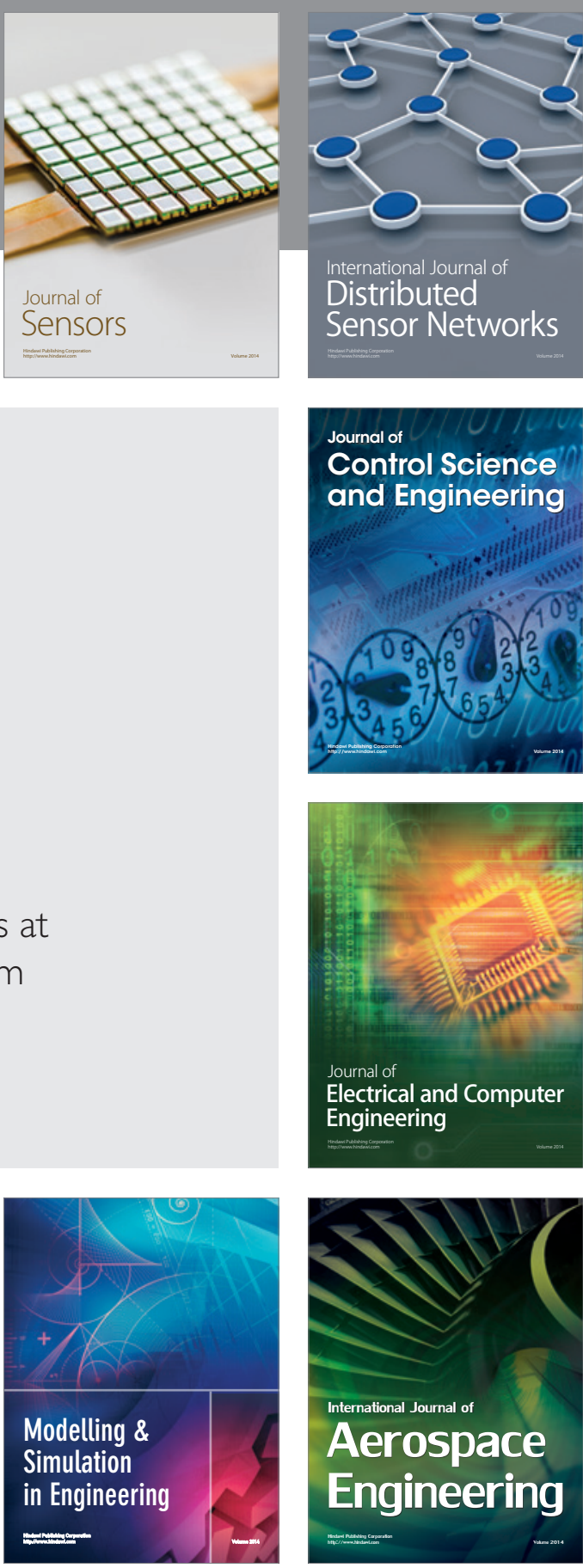

International Journal of

Distributed

Sensor Networks

Journal of

Control Science

and Engineering
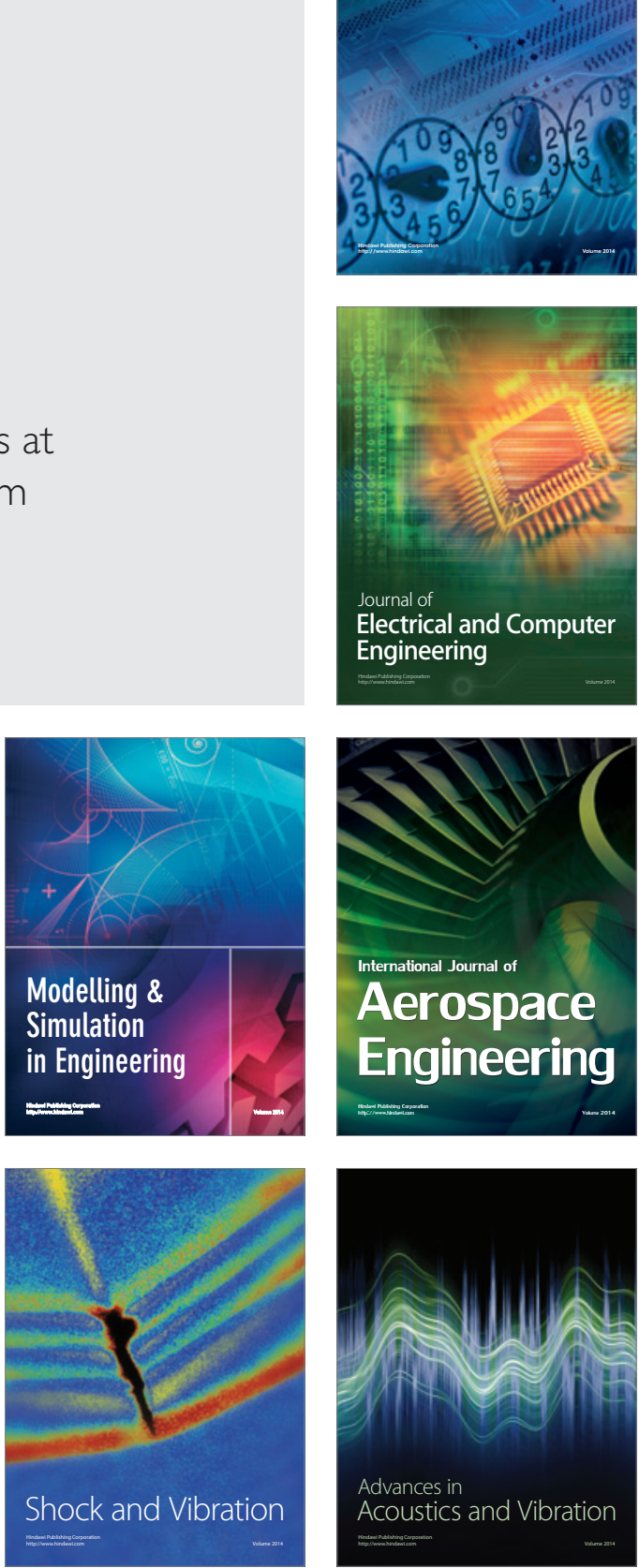